\title{
Rating the Customer Requirements based on DEMATEL and Entropy
}

\author{
http://dx.doi.org/10.3991/ijoe.v9iS4.2590 \\ Yang Mingshun, Yu Ting, Liang Yanjie and Zhang Yubo \\ Country Xi'an University of Technology, Xi' an, China
}

\begin{abstract}
In the configuration procedure of QFD Quality Function Deployment, the importance rating of customer requirements as one of the important input parameters is very significant to the determination of the technical characteristics and even to the optimum decision to the whole quality of house as well as the following step of allocation decision-making. In this paper, firstly the drawbacks of the existing methods of determining the importance rating of customer requirements are analyzed. Then with the influence of customer requirement interdependence and market competitive evaluations sufficiently considered, DEMATEL is introduced to deal with self-correlations between customer requirements and Entropy is used to dispose the market competitive evaluations to modify the fundamental importance ratings of the customer requirements, thus a more objective comprehensive fundamental importance rating of the customer requirements can be gained. Finally, one example is given to verify the effectiveness of the presented method.
\end{abstract}

Index Terms-DEMATEL, entropy, importance rating of customer requirements

\section{INTRODUCTION}

With the development of manufacturing globalization \& networking and information technology, higher customer satisfaction, lower cost and shorter product development cycle has become key to gain a competitive advantage. Quality Function Deployment (QFD) as a structured user-driven, systematic product development and quality assurance method is gained more and more concerns [1]. Numerous facts show that, the correct implementation of QFD can effectively help the enterprises to improve product quality and customer satisfaction, reduce cost and shorten product life cycle [2].

QFD generally includes two basic processes, i.e. the extraction of customer requirements and the waterfall-type decomposition process of customer requirements [3]. Among them, the importance of customers' requirements is an important input in the building process of house of quality (HoQ). It plays an important role to determine the technical characteristics and the optimum of the house of quality and subsequent stages of the allocation decisionmaking; it is also very important to the characteristics of the products and parts and continued production control. Based on importance rating, manufacturers can design and develop products purposefully to make customer satisfied in order to gain a competitive advantage [4][5]. Therefore, in the process of building product planning HoQ, it is one of the key works to access to customer requirements and importance ratings [6].
Firstly, a general procedure of determining the importance of customer requirements in the product planning HoQ is analyzed and the deficiencies of the existed methods are pointed out. Then considering the influence of customer requirement correlations and market competitive evaluations to the customer requirements, a correctional method based on the fundamental importance of customer requirements is presented. Finally, application study is given to illustrate the presented method.

\section{DETERMINATION OF IMPORTANCE RATING OF Customer ReQuirements in Product Planning HoQ}

The importance rating of customer requirements in the product planning is the fundamental input for the house of quality. Generally, its determination refers to the following steps [7].

(1) Determining the $m$ customer requirements for the given product by kinds of surveying methods, it can be written as $C A=\left(C A_{1}, \cdots, C A_{m}\right)$.

(2) Obtaining the fundamental importance rating of the obtained customer requirements by AHP or other methods: $w_{o}=\left(w_{o}^{1}, \cdots, w_{o}^{m}\right)$.

(3) Making competitive evaluation among the similar products in the market. Here, $C_{1}$ represents for the considered enterprise, while $C_{2}, \cdots, C_{k}$ for the competitive enterprises. With market survey for customer requirements carried out, the market competitive assessment matrix can be obtained as

$$
\begin{aligned}
& Z=\left\{z_{j i} \mid j=1, \cdots, m, i=1, \cdots, k\right\}:
\end{aligned}
$$

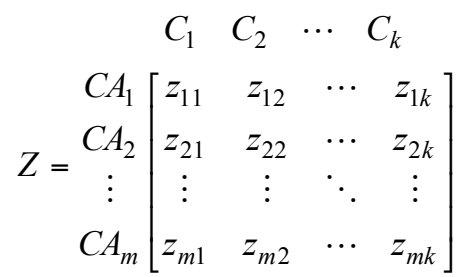

Based on Z, information for modifying the importance rating of customer requirements can be obtained based on market competitive evaluations: $w_{s}=\left(w_{s}^{1}, \cdots w_{s}^{m}\right)$.

(4) By integrating with the fundamental importance rating of customer requirements $w_{o}$ and the modifying market based on the competitive evaluations $w_{s}$, the final importance ratings of customer requirements can be obtained as $f=w_{o} \times w_{s}$ or $f=w_{g} w_{o}+w_{e} w_{s}, w_{g} \geq 0$, 
$w_{e} \geq 0, \quad w_{e}+w_{g}=1, j=1, \cdots, m$. While using the linear synthesis to obtain the final importance ratings of customer requirements, $w_{g}$ and $w_{e}$ separately represent for the compromised weight of the fundamental importance rating of customer requirements and the market competitive evaluations modification.

Among them, the fundamental importance rating of customer requirements is usually obtained by surveying or AHP or ANP and other integrated methods [5-8]. For the market competition assessment information, in the majority of studies the concept of 'sale point' were subjectively introduced to modify the fundamental importance rating of customer requirements, in this way information of the fundamental importance rating of customer requirements were double-counted, therefore, they can not reflect the impact of the competitive assessment on the importance of customer requirements. At the same time, in the process of building house of quality, the influence of technical characteristics correlations is taken in fully considerations while the influence of customer requirement correlations is insufficient. In fact, while customers express their requirements, different requirements often influence each other. With a CD player, for example, with the development of the society, customer's requirement on security is becoming much higher. Security of a CD player will have positive effect on its compatibility, so they are positively correlated. While high security requires higher cost, which will result in a high price, so security and price are of negative correlation. The correlation among the requirements will make a requirement with lower basic importance need be given more attention, improving the requirement will indirectly improve other requirements. In the same way, for a requirement with higher basic importance, with the correlation considered, its importance will become lower. Thus, to obtain a more accurate customer requirement importance, the correlation among the requirements should be taken into consideration. To obtain more accurate information of importance rating of customer requirements, in this paper, the influence of customer requirement correlations and market competitive evaluations to correct the fundamental importance rating of customer requirements are synthetically considered.

\section{MODIFYING THE FUNDAMENTAL IMPORTANCE RATING OF CUSTOMER REQUIREMENTS WITH CONSIDERATION OF CUSTOMER REQUIREMENT CORRELATIONS}

The decision making trial and evaluation laboratory (DEMATEL) is a useful methodology to visualize the structure of complicated causal relationships [9][10]. Unlike the traditional techniques such as analytic hierarchy process with the assumption that elements are independent, this method, one of the structural modeling techniques, can identify the interdependence among the elements of a system through a causal diagram, which is shown in the Fig.1. It uses graph theory and matrix tool for system analysis and can modify the corresponding factors' weights according to the inter-influence among the factors. In the method, through the various factors' logical relationship and the direct impact matrix, how one factor affects another and how one factor is affected by another can be obtained.

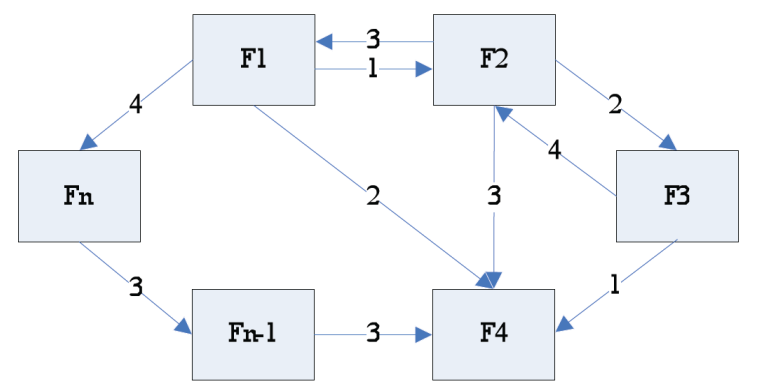

Figure 1. Mutual influence of a directed graph

The DEMATEL method can be summarized in the following steps:

(1) Determine the impact factors of the system.

(2) Analyze the relationship among the factors, construct directed graphs and indicate the strength of the relationship between the factors by numbers. Evaluate the direct influence between any two factors by an integer score ranging from $0,1,2$, and 3 , representing 'no influence', 'low influence', 'medium influence', and 'high influence', respectively.

(3) Express the above contents into matrix form which is called direct impact matrix $\mathrm{Y}=\left[y_{i j}\right]_{\mathrm{N} \times \mathrm{N}}$, the notation of $y_{i j}$ indicates the degree to which the respondent believes factor $i$ affects factor $j$. For $i=j$, the diagonal elements are set to zero.

(4) Standardize the direct impact matrix $Y$, and the standardized impact matrix $\mathrm{X}$ will be obtained.

(5) Based on the standardized impact matrix $X$, the overall impact matrix $\mathrm{T}$ can be obtained, namely crossreinforcement matrix from the following formula.

$$
T=X(I-X)^{-1}=T\left(t_{i j}\right)_{n \times n}
$$

Where $t_{i j}$ represents for direct and indirect affecting degree of the factor $i$ and $j$.

As mentioned above, in product planning HoQ, the different customer requirements usually affects each other, moreover, the intensity of impact may be different. In DEMATEL, the causal diagram uses digraphs rather than directionless graphs to portray the basic concept of contextual relationships and the strengths of influence among the elements. Thus it can well interpret the interaction relationship among the customer requirements. In this paper, firstly the causal diagram of customer requirements is obtained through the DEMATEL, then it is analyzed, the cross-reinforcement matrix $\mathrm{T}$ can be obtained, finally the fundamental importance rating of customer requirements are modified.

\section{IV.MODIFYING THE FUNDAMENTAL IMPORTANCE RATING OF CUSTOMER REQUIREMENTS WITH CONSIDERATION OF MARKET COMPETITIVE EVALUATION}

In information theory, entropy indicates the amount of information obtained from a group of uncertain things. For the amount of information contained in a discrete probability distribution, the greater the distribution changes the more abundant information it contains [11][12]. Shannon defines this information entropy for measuring the amount of information as: 


$$
E\left(p_{1}, \cdots, p_{k}\right)=-\varphi_{k} \sum_{i=1}^{k} p_{i} \ln \left(p_{i}\right)
$$

Where, $\varphi_{k}=\frac{1}{\ln (k)}$, it can be seen that the bigger $E\left(p_{1}, \cdots, p_{k}\right)$ is, the less information $p_{1}, \cdots, p_{k}$ contains.

In QFD, competitive assessment refers to a satisfaction evaluation for similar products of different manufacturers on the market from the perspective of the customers to reflect the strengths and weaknesses of existing product as well as improvement needed to be done in emergency. According to the assessment of market competition for customer requirements, if the customer's assessment is consistent, it means the product homogeneity is comparatively strong for the given customer requirement, which implies that improvement of the requirement will likely significantly improve customer's satisfaction. Thus the customer's requirement can be given a higher attention. If the customer's assessment of competing products requirements more dispersed, it indicates that the market requirement for products has obvious heterogeneity. It is difficulty to enhance customer's satisfaction by improving the requirement. Therefore, a lower attention should be taken into consideration.

The above analysis shows that the concept of entropy is very consistent with the extracted information through the market competitive evaluation of customer requirements, therefore in this article introduces the concept of entropy to get the importance rating of customer requirements correction through dealing with the information of market competitive evaluation.

\section{FinAL DETERMINATION OF CUSTOMER REQUIREMENTS IMPORTANCE RATINGS}

Firstly, DEMATEL method is used to analyze the interdependence among the customer requirements and the obtained cross-reinforcements matrix is used to modify the basic importance of customer requirements. Then based on the concept of entropy, market competitive evaluations are dealt to modify the importance rating of customer requirements, finally the final importance ratings can be obtained. The corresponding steps are as follows:

(1) Determine the interaction digraph of customer requirements and establish a direct influence matrix $\mathrm{Y}$ and standardized matrix $X$.

(2) According to formula (1) to get the total impact matrix $\mathrm{T}$ of customer requirements.

(3) Take the total impact matrix $\mathrm{T}$ as a cross- reinforcement matrix. Modify the importance rating of CRs, i.e.

$$
w_{T}^{j}=\sum_{i=1}^{n} t_{i j} w_{o}^{i} / \sum_{i=1}^{n} \sum_{j=1}^{n} t_{i j} w_{o}^{i}
$$

(4) For customer needs $C A_{j}$, the results of the market competitive evaluations is $z_{j 1}, \cdots, z_{j k}$, let $z_{j}=\sum_{i=1}^{k} z_{j i}$, taking $p_{j i}=z_{j i} / z_{j}$ as a discrete probability distribution sub-sample of $k$ manufacturers about $C A_{j}$, thus the evaluation information entropy about $C A_{j}$ can be defined

$$
\begin{gathered}
E\left(p_{1}, \cdots, p_{k}\right)=-\varphi_{k} \sum_{i=1}^{k} p_{i} \ln \left(p_{i}\right)=-\varphi_{k} \sum_{i=1}^{k}\left(z_{j i} / z_{j}\right) \ln \left(z_{j i} / z_{j}\right) \\
w_{S}^{j}=E\left(C A_{j}\right) / \sum_{j=1}^{m} E\left(C A_{j}\right)
\end{gathered}
$$

Calculate the final importance ratings of the customer requirements $f_{j}$

$$
f_{j}=\alpha w_{T}^{j}+(1-\alpha) w_{S}^{j}
$$

$\alpha$ is the scale factor, it reflects the tradeoffs between interdependence among the customer requirements and the market competitive evaluation, its value can be determined according to the actual situation of the product development, usually $\alpha$ can be equal to 0.5 .

\section{VI.APPLICATION STUDY}

Being known 8 customer requirements of $\mathrm{CD}$ player: $C A=\left(C A_{1}, \cdots, C A_{8}\right)=$ (High definition, nice sound, easy to use, portability, stylish, safe and reliable, compatibility, affordable), the fundamental importance rating of customer requirements is $w_{o}=\left(w_{o}^{1}, \cdots, w_{o}^{8}\right)=(0.4,0.2,0.8,0.74$, $0.34,0.8,0.54,0.8)$. DEMATEL method is used to analyze the interdependence among the customer requirements, which is shown in the Fig.2.

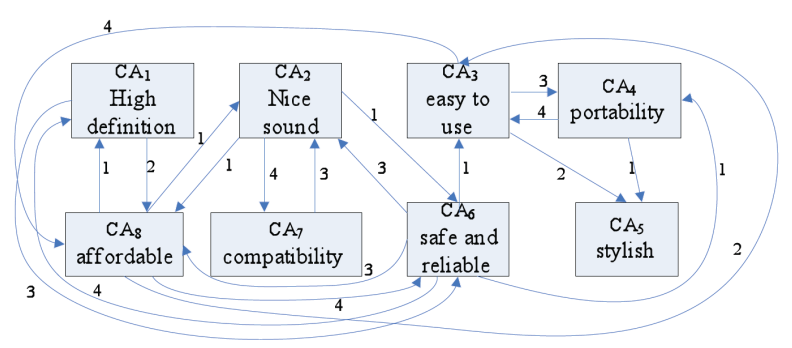

Figure 2. Interaction digraph of customer requirements

Quantify the interaction digraph of customer requirements and establish a direct influence matrix $\mathrm{Y}$ and standardized matrix $\mathbf{X}$.

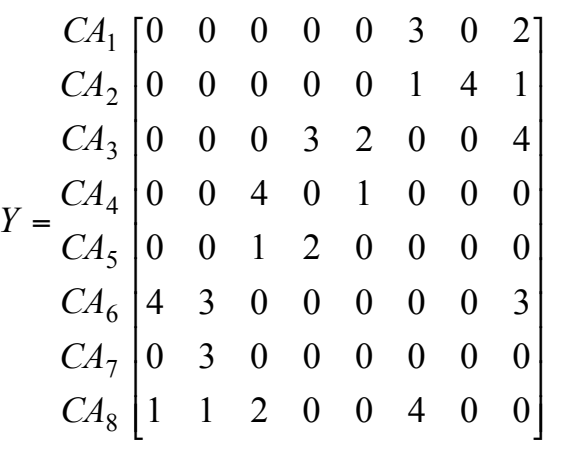




$$
X=\left[\begin{array}{cccccccc}
0 & 0 & 0 & 0 & 0 & 0.3 & 0 & 0.2 \\
0 & 0 & 0 & 0 & 0 & 0.1 & 0.4 & 0.1 \\
0 & 0 & 0 & 0.3 & 0.2 & 0 & 0 & 0.4 \\
0 & 0 & 0.4 & 0 & 0.1 & 0 & 0 & 0 \\
0 & 0 & 0.1 & 0.2 & 0 & 0 & 0 & 0 \\
0.4 & 0.3 & 0 & 0 & 0 & 0 & 0 & 0.3 \\
0 & 0.3 & 0 & 0 & 0 & 0 & 0 & 0 \\
0.1 & 0.1 & 0.2 & 0 & 0 & 0.4 & 0 & 0
\end{array}\right]
$$

(2) According to formula (1) to get the total impact matrix $\mathrm{T}$ of customer requirements.

$$
T=\left[\begin{array}{llllllll}
0.3048 & 0.2748 & 0.1260 & 0.0437 & 0.0296 & 0.6301 & 0.1099 & 0.5279 \\
0.1333 & 0.2573 & 0.0615 & 0.0213 & 0.0144 & 0.2687 & 0.5029 & 0.2576 \\
0.2243 & 0.2118 & 0.3671 & 0.4743 & 0.3209 & 0.3791 & 0.0847 & 0.7266 \\
0.0938 & 0.0886 & 0.5720 & 0.2188 & 0.2363 & 0.1586 & 0.0354 & 0.3040 \\
0.0412 & 0.0389 & 0.2511 & 0.2912 & 0.0793 & 0.0696 & 0.0156 & 0.1335 \\
0.7028 & 0.6202 & 0.1779 & 0.0617 & 0.0417 & 0.5709 & 0.2481 & 0.7450 \\
0.0400 & 0.3772 & 0.0184 & 0.0064 & 0.0043 & 0.0806 & 0.1509 & 0.0773 \\
0.4698 & 0.4436 & 0.3633 & 0.1261 & 0.0853 & 0.7941 & 0.1775 & 0.5219
\end{array}\right]
$$

The comprehensive impact matrix as a crossreinforcement matrix, use formula (2) to get the effect weighting of customer requirements $(0.1273,0.0846$, $0.1771,0.1116,0.0622,0.1749,0.0312,0.1792)$

Using entropy to analyze the market competitive evaluations of the 5 factories. The market competitive evaluations matrix according to the 8 requirements is:

$$
Z=\left[\begin{array}{lllll}
0.220 & 0.180 & 0.200 & 0.275 & 0.325 \\
0.480 & 0.520 & 0.550 & 0.500 & 0.575 \\
0.720 & 0.320 & 0.375 & 0.425 & 0.225 \\
0.480 & 0.740 & 0.700 & 0.500 & 0.750 \\
0.460 & 0.620 & 0.350 & 0.500 & 0.550 \\
0.300 & 0.520 & 0.515 & 0.485 & 0.625 \\
0.800 & 0.580 & 0.325 & 0.500 & 0.750 \\
0.520 & 0.320 & 0.425 & 0.275 & 0.300
\end{array}\right]
$$

Using formula (4), (5) obtains the competitive evaluations information entropy and normalization: $(0.1257$, $0.1274,0.1215,0.1261,0.1263,0.1255,0.1224,0.1252$ )

(5) Using formula (6), taking $\alpha=0.5$, then in the final importance ratings of the customer requirements are : $(0.1265,0.1060,0.1493,0.1189,0.0943,0.1502,0.0768$, $0.1522)$

(6) Rank the customer requirements according to the importance rating from high to low: CA8, CA6, CA3, CA1, CA4, CA2, CA5, CA7.

In this application, the initial importance orders of CAs are: $\mathrm{CA}_{3}, \mathrm{CA}_{6}, \mathrm{CA}_{8}, \mathrm{CA}_{4}, \mathrm{CA}_{7}, \mathrm{CA}_{1}, \mathrm{CA}_{5}, \mathrm{CA}_{2}$, while with the interdependence among the $\mathrm{CAs}$ and market competition analysis considered, the importance orders are: $\mathrm{CA}_{8}, \mathrm{CA}_{6}, \mathrm{CA}_{3}, \mathrm{CA}_{1}, \mathrm{CA}_{4}, \mathrm{CA}_{2}, \mathrm{CA}_{5}, \mathrm{CA}_{7}$, which shows that obvious changes occur in the ordering..

\section{CONCLUSION}

In this paper, based on the basic customer requirements importance, with influence of correlation among the CAs and market competition analysis on the final importance of the CAs considered, DEMATEL and entropy are introduced to deal with the corresponding information to updated the customer requirements importance, and the modified importance are aggregated to obtain the final importance ratios, which makes determination of the customer requirement importance more objective and reasonable. The application study shows that with the correlations among the CAs considered, the importance ratios of the CAs are updated, and the corresponding importance ordering change obviously.

\section{ACKNOWLEDGMENTS}

This work was supported by the National Natural Science Foundation, China project [60903124], the Doctorial Program Foundation of Higher Education of China project [20096118120003], the Science and Technology Research Plan of Shannxi Provincial Education Department, China project [09JK614] and the Science and Technology Planning Project of Xi'an, China project [CX1255-4].

\section{REFERENCES}

[1] L. K. Chan, and M. L. Wu, "Quality Function Deployment: a Literature Review", European Journal of Operational Research, vol. 143, no. 6, pp. 463-497, December, 2002. http://dx.doi.org/10.1016/S0377-2217(02)00178-9

[2] L. H. Lin, H. P, Cheng, and M. L. Tseng et al, "Using QFD and ANP to Analyze the Environmental Production Requirements in Linguistic Preferences", Expert Systems with Applications, vol. 37, no. 3, pp. 2186-2196, March 2010. http://dx.doi.org/10.1016/ j.eswa.2009.07.065

[3] H. T. Liu, "The Extension of Fuzzy QFD: From Product Planning to Part Deployment", Expert Systems with Applications", vol. 36, no. 8, pp. 11131-11144, October, 2009. http://dx.doi.org/10.1016/ j.eswa.2009.02.070

[4] H. T. Liu, and C. H. Wang, "An Advanced Quality Function Deployment Model Using Fuzzy Analytic Network Process", Applied Mathematical Modelling, vol. 34, no. 11, pp. 3333-3351, November, 2010. http://dx.doi.org/10.1016/j.apm.2010.02.024

[5] Y. L. Li, J. F. Tang, and X. G. Luo, "An ECI-based Methodology for Determining the Final Importance Ratings of Customer Requirements in MP Product Improvement ", Expert Systems with Applications, vol. 37, no. 9, pp. 6240-6250, September, 2010. http://dx.doi.org/10.1016/j.eswa.2010.02.100

[6] C. K. Kwong, and H. Bai, "A Fuzzy AHP Approach to the Determination of Importance Weights of Customer Requirements in Quality Function Deployment ", Journal of Intelligent Manufacturing, vol. 13, no. 5, pp. 367-377, October, 2002. http://dx.doi.org/10.1023/A:1019984626631

[7] M. S. Yang, and Z. H Lin, "Method to Determine Importance of Customers' Requirements in QFD ", Journal of management sciences, vol. 6, no. 5, pp. 65-71, October, 2003.

[8] Z. J. Kong, and Y. J. Hao, "Determine the Importance of Customer Requirement in QFD Using Importance - Probability Comprehensive Coefficient Method", Computer Integrated Manufacturing System, vol. 7, no. 2, pp. 65-67, July, 2001.

[9] Y. K Liang, and Z. D. Ma, "Construction Risk Analysis of Foundation Engineering Based on DEMATEL Method ", Journal of Engineering Management, vol. 24, no. 2, pp. 161-164, February, 2010 .

\section{AUTHORS}

YANG Mingshun received the B.S. degree and M.S. degree from Yanshan University (YSU), Qinhuangdao, China in 1995 and 1998 respectively. He received the $\mathrm{PhD}$. degree in Mechanical Engineering from Xi'an Jiaotong University (XJTU), Xi'an, China. He is currently an associate professor in the Department of Mechanical 
Manufacturing and Automation, School of Mechanical and Precision Instrument Engineering, Xi'an University of Technology (XAUT), Xi'an, China. He has authored/coauthored over 30 publications and two book chapters. His research interests include production system designing and planning, integrated quality management and advanced manufacturing technology. (e-mail: yangmingshun@xaut.edu.cn)

YU Ting was born in Shaanxi Province, China. She is currently a M.S. student in School of Mechanical and Precision Instrument Engineering, Xi'an University of Technology (XAUT), Xi'an, China. She received his B.S. degree in Industrial Engineering in 2011 from XAUT. Her research interests include reliability and quality control. (e-mail: nocc@163.com)

Liang Yanjie was born in Shaanxi Province, China. She is currently a M.S. student in School of Mechanical and Precision Instrument Engineering, Xi'an University of Technology (XAUT), Xi'an, China. She received his B.S. degree in Industrial Engineering in 2011 from XAUT. Her research interests include reliability and quality control. (e-mail: ccyxz@xaut.edu.cn).

ZHANG Yubo was born in Shaanxi Province, China. He is currently a M.S. student in School of Mechanical and Precision Instrument Engineering, Xi'an University of Technology (XAUT), Xi'an, China. He received his B.S. degree in Industrial Engineering in 2009 from XAUT. His research interests include reliability and quality control. (e-mail: yancici@yeah.net).

This article is an extended and modified version of a paper presented at the International Conference on Mechanical Engineering, Automation and Material Science (MEAMS2012), held 22-23 December 2012, Wuhan, China. Manuscript received 18 March 2013. Published as resubmitted by the authors 01 May 2013. 